\title{
QT Interval Prolongation during Rapid Fall in Blood Glucose in Type I Diabetes
}

\author{
TF Christensen ${ }^{1,2}$, I Lewinsky ${ }^{1}$, LE Kristensen ${ }^{2}$, J Randløv ${ }^{2}$, JU Poulsen ${ }^{2}$, \\ E Eldrup $^{3}$, C Pater $^{2}$, OK Hejlesen ${ }^{1}$, JJ Struijk ${ }^{1}$ \\ ${ }^{1}$ Aalborg University, Aalborg, Denmark \\ ${ }^{2}$ Novo Nordisk A/S, Hillerød, Denmark \\ ${ }^{3}$ Steno Diabetes Center, Gentofte, Denmark
}

\begin{abstract}
Prolongation of QT interval on the ECG has been shown to be possibly associated with hypoglycaemia. In this study we investigated QT prolongation in episodes of single bolus induced hypoglycaemia in ten subjects with known type 1 diabetes mellitus.

A mean QTc prolongation from baseline of 27(SD 19) ms $(p<0.001)$ was measured 15 minutes after the injection of insulin. At this point the mean blood glucose was 7.2(SD 3.1) $\mathrm{mmol} / \mathrm{L}$. At the nadir of blood glucose the mean QTc prolongation from baseline was 25 (SD 22) $m s(p<0.001)$.

The study suggests that changes in the QTc in diabetics may occur not only as a result of low blood glucose per se but maybe also during rapid fall in blood glucose. The finding could be explained by pathophysiological changes in diabetes.
\end{abstract}

\section{Introduction}

There is considerable evidence that during insulininduced hypoglycemia there is an altered repolarization of the cardiac cells notable on a surface ECG in both healthy [1-6] and diabetic subjects [7-9]. The altered repolarization is seen in the ECG as a flattened and prolonged $\mathrm{T}$ wave and in some cases the development of a U wave resulting in a prolonged QT interval (corrected for heart rate - QTc) [8]. Fatal cardiac arrythmia caused by hypoglycaemia-induced QT prolongation has been hypothesized to be the cause of at least some of the incidents of the dead-in-bed syndrome [8] in young, otherwise healthy, type I diabetes patients [10].

There are two mechanisms hypothesized to be responsible for the QT prolongation: the sympathoadrenal activation as part of the glucose counterregulation and hypokalemia caused by elevated levels of adrenaline and insulin during hypoglycaemia [11]. Robertsen et al. [11] showed that during a hyperinsulinemic clamp QT prolongation in healthy subjects developing hypoglycaemia was prevented by beta blockade but only slightly reduced by clamping potassium. This result indicates that the sympathoadrenal response to hypoglycemia may be the cause of QT prolongation rather than being caused by hypokalemia.

The complex mechanism of QT prolongation during hypoglycaemia is however far from being understood completely. Only few studies investigating the QT prolongation during hypoglycaemia have been conducted on diabetes patients limiting the applicability of the results. Diabetes is a metabolic syndrome which specifically involves disturbances in the hormones responsible for the QT prolongation, insulin and adrenaline. Glucose counterregulation is known to be altered in persons with diabetes [12] and this might produce different alterations in the QT interval as compared to healthy subjects. The hyperinsulinemic clamp procedure is frequently used when inducing hypoglycaemia experimentally to explore changes in the QT interval and while this procedure ensures homogeneous blood glucose curves it is quite invasive and presents a non-physiological situation.

In this study we investigated the QT prolongation during insulin-induced hypoglycaemia in type 1 diabetes subjects with the main objective to determine start of and pattern of QT prolongation.

\section{Methods}

We studied 10 subjects with type 1 diabetes (C-peptide negative). Subjects had $\mathrm{HbA1c}<10 \%$ and none had signs of neuropathy. Other clinical details can be seen in table 1.

Each subject attended the clinic two weekends separated by at least 4 weeks. At each weekend the subject had induced hypoglycemia two times; Saturday at 
02:00 and 22:00. Subjects were randomized to use either insulin aspart (NovoRapid, Novo Nordisk A/S, Denmark) or human insulin (Actrapid, Novo Nordisk A/S, Denmark) the first weekend in a cross-over design so the other type of insulin was used at the next visit.

Subjects had a catheter (Venflon, Viggo AB, Sweden) inserted in to the right-hand dorsal vein for sampling of blood and administration of insulin and glucose.

Tabel 1. Clinical details of the diabetic subjects. Results for age, BMI and diabetes duration are given as mean \pm SD.

\begin{tabular}{lc}
\hline \hline Number & $10(6$ men +4 women $)$ \\
Age & $32 \pm 9$ years \\
BMI & $25 \pm 2 \mathrm{~kg} / \mathrm{m}^{2}$ \\
Diabetes Duration & $15 \pm 10$ years \\
\hline \hline
\end{tabular}

Hypoglycemia was induced by a single bolus of insulin $(0.15 \mathrm{u} / \mathrm{kg}$ bodyweight $)$ injected via the catheter. Blood glucose was measured (HemoCue Glucose 201 DM, Ängelholm, Sweden) 30 minutes prior to insulin injection and every 5 minutes following the injection. When blood glucose reached $3 \mathrm{mmol} / \mathrm{l}$, samples were taken every 2 minutes until a blood glucose of $2.4 \mathrm{mmol} / 1$ was reached and intravenous glucose $(10 \%)$ was administered to restore the blood glucose.

\subsection{ECG measurements}

ECG was recorded by disposable $\mathrm{Ag} / \mathrm{AgCl}$ electrodes (Blue Sensor L, Ambu A/S, Denmark) in the Einthoven lead II configuration and sampled at $400 \mathrm{~Hz}$ with a 12 bit resolution using a data acquisition system (Portilab 16+2, Twente Medical Systems International, Holland).

One minute of baseline ECG was recorded 30 minutes prior to insulin injection and the sampling continued to 60 minutes after the blood glucose nadir. ECG was processed using software developed in MatLab version 7.4 (Mathworks, USA). Data from each session was filtered with a low pass filter adapting to the heart rate so baseline wander was suppressed. R-peaks were found using a matched filtering technique and median beats were obtained from one minute windows, giving between 40 and 90 beats for each median. The median beat was discarded if the estimated signal-to-noise ratio was below $20 \mathrm{~dB}$.

\subsection{QT interval measurements}

QT interval measurements were carried out on each median beat using a fully automatic computer algorithm based on the tangent approach. The T-wave was high pass filtered to get a smooth curve before a tangent was fitted to the point of maximum gradient at the downward slope of the T-wave. The end of the T-wave was defined as the intersection between this tangent and the isoelectric level found in the PQ-interval. QTc was calculated using Fridericias formula $\left(\mathrm{QT} / \mathrm{RR}^{1 / 3}\right)$.

\subsection{Data analysis}

Of the 40 recorded episodes of hypoglycemia, 8 were excluded, leaving 32 episodes to further analyses. Six episodes were excluded because the subject had hypoglycemia during baseline at 30 minutes before the intended insulin injection. Two episodes were excluded because of instrumentation issues. A minimum of two episodes from each subject were used in the analysis

Data are given as mean $\pm \mathrm{SD}$ and a paired t-test was used to test for significant QTc changes compared to baseline measurements across all recorded episodes with $\mathrm{p}<0.01$ considered significant.

\section{Results}

The time from insulin injection to hypoglycaemia was $45 \pm 32$ minutes. No significant difference between the two

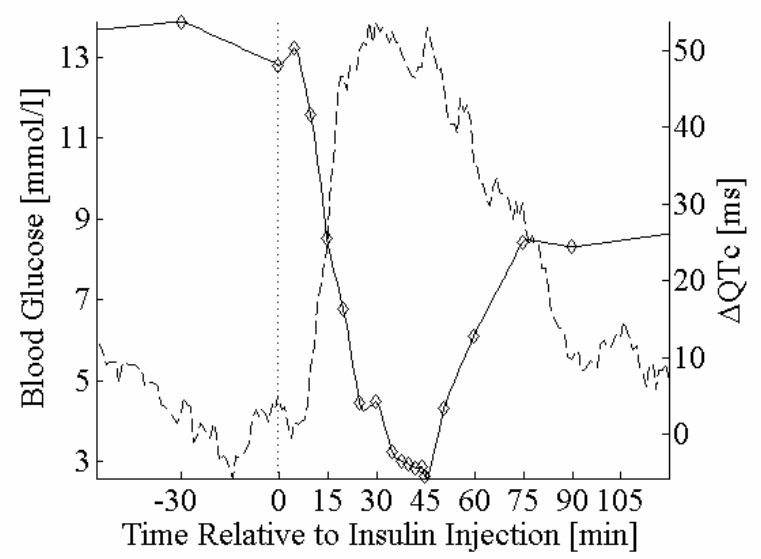

Figure 1. The development of blood glucose (solid line) and the change in QTc from baseline $(\triangle \mathrm{QTc}-$ dashed line) during an episode of hypoglycemia in a male diabetic subject. The vertical dotted line marks the time of insulin injection and diamonds indicate blood glucose measurements.

types of insulin with respect to QTc prolongation or time from insulin injection to hypoglycaemia was observed. 
Tabel 2. The table shows the changes in QTc from baseline, blood glucose and rate of change of blood glucose at insulin injection, 15 and 30 minutes after injection as well as at the BG nadir. Results are shown as mean \pm SD. Numbers marked with ' $*$ ' indicates a statistical significant difference from baseline $(\mathrm{p}<0.001)$.

\begin{tabular}{lcccc}
\hline & $\begin{array}{c}\text { Insulin } \\
\text { Injection }\end{array}$ & +15 min & +30 min & BG Nadir \\
\hline QTc Change from Baseline [ms] & $-0 \pm 5$ & $27 \pm 19^{*}$ & $25 \pm 22^{*}$ & $25 \pm 24^{*}$ \\
Blood Glucose [mmol/1] & $10.5 \pm 3.7$ & $7.2 \pm 3.1^{*}$ & $5.4 \pm 2.9^{*}$ & $2.3 \pm 0.2^{*}$ \\
$\begin{array}{l}\text { Rate of Change in Blood } \\
\text { Glucose [mmol/1/min] }\end{array}$ & $-0.00 \pm 0.08$ & $-0.34 \pm 0.17^{*}$ & $-0.14 \pm 0.17^{*}$ & 0 \\
\hline \hline
\end{tabular}

Significant QTc prolongation was present shortly after the injection of insulin as is evident from figure 1, figure 2 and table 2. 15 minutes after insulin injection, where the subjects were normoglycemic with a mean blood glucose of $7.2 \pm 3.1 \mathrm{mmol} / \mathrm{l}$, a significant QTc prolongation of $27 \pm 19 \mathrm{~ms}(\mathrm{p}<0.001)$ was measured. At the blood glucose nadir where the subjects where hypoglycemic (blood glucose $2.3 \pm 0.2 \mathrm{mmol} / \mathrm{l}$ ) the QTc prolongation was $25 \pm 24$ ms $(p<0.001)$. The difference between QTc at $15 \mathrm{~min}$ after injection and at BG nadir was not significant $(\mathrm{p}=0.25)$.

The rate of change in blood glucose was at $15 \mathrm{~min}$ after injection $-0.34 \pm 0.17 \mathrm{mmol} / 1 / \mathrm{min}$ and significantly higher than at insulin injection $(\mathrm{p}<0.001)$.

\section{Discussion and conclusions}

In this study the dynamics of changes in the QT interval were examined during hypoglycaemia induced by a single bolus of insulin. The study was performed to investigate the dynamics of QT interval changes during hypoglycaemia in diabetic subjects as this area is not very well documented.

It is evident from the results of this study that prolongation of the QT interval occurs not only as a result of hypoglycaemia per se. The measured prolongation of QTc during hypoglycemia $(2.3 \mathrm{mmol} / \mathrm{l})$ is in fact not significantly different from the prolongation $15 \mathrm{~min}$ after insulin injection where the blood glucose was euglycemic $(\mathrm{BG}=7.2 \pm 3.1 \mathrm{mmol} / \mathrm{l})$. Based on previous findings in the literature we expected that the QTc would be significantly different at hypoglycaemia as compared to normoglycemia.

The QT prolongation during hypoglycaemia has previously been attributed to the counterregulatory hormones and namely adrenaline $[8,11]$. The release of adrenaline is however first triggered when the subject reaches hypoglycaemia and thus does not explain the prolongation we see at fast fall normoglycemia.

An explanation of why the prolongation is present at normoglycemia could be the high concentrations of

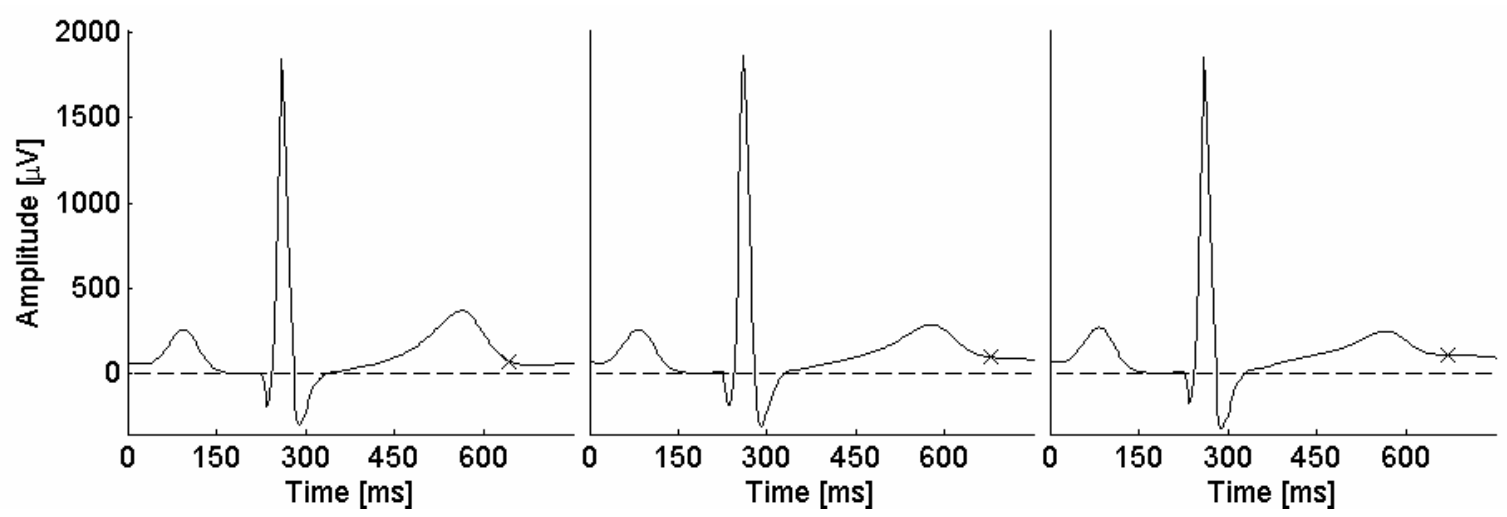

Figure 2. Three median beats from a male diabetic subject at baseline (left), $15 \mathrm{~min}$ after injection (middle) and during hypoglycemia (right). The ' $x$ ' marks the end of the T-wave. Notice the pronounced flattening and lengthening of the Twave and the similarity between the beat during fast fall normoglycemia (middle) and hypoglycemia (right). The ' $x$ ' marks the end of the T-wave. 
insulin following the iv. bolus. Gastaldelli et al. [2] showed that hyperinsulinemic normoglycemia triggered a modest QTc prolongation of $8 \mathrm{~ms}$. Thus a part of the prolongation seen in this study could be attributed to the high insulin concentrations. The QTc lengthening effect of insulin is mainly attributed to the lowering effect of the serum potassium concentration causing hypokalemia.

A difference between this study and most other studies investigating QT prolongation during hypoglycemia is the use of diabetic subjects. Diabetes is a metabolic syndrome which affects the key hormones, insulin and adrenaline, responsible for the QT prolongation seen during hypoglycemia. It is plausible that the effect of adrenaline and insulin and thus the dynamics of QTc prolongation are altered in the diabetic subject compared to the healthy subject. DeFronzo et al. [12] showed counterregulatory responses caused by a fall in blood glucose from 11 $\mathrm{mmol} / \mathrm{l}$ to $6 \mathrm{mmol} / \mathrm{l}$ in diabetic subjects. This observation is to some extend supported by this study if QTc prolongation is seen as an indirect marker sympathoadrenal activation. In this study the fasting blood glucose was $10.5 \pm 3.7 \mathrm{mmol} / \mathrm{l}$ and a counterregulatory response could thus be expected before reaching hypoglycemic blood glucose levels. It is not possible to conclude from this study if the prolongation at normoglycemia is caused be the falling blood glucose or a higher level at which the counterregulation is initiated.

Thus the results from this study indicate that QT interval prolongation in diabetes might not be limited to low blood glucose, but is experienced also when the blood glucose is declining after an insulin injection. Additional studies need to clarify if the changes are clinically relevant and if the same results are obtainable when the insulin is given subcutaneously.

\section{Acknowledgements}

This study has been supported by Novo Nordisk A/S. We are grateful to all the patients, volunteers and clinical staff who took part in the study.

Address for correspondence

Toke Folke Christensen

Aalborg University

Frederik Bajers Vej 7D1

DK-9210 Aalborg

tofc@hst.aau.dk

\section{References}

[1] Eckert B, Agardh CD. Hypoglycaemia leads to an increased QT interval in normal men. Clinical Physiology 1998;18;570-575

[2] Gastaldelli A, Emdin M, Conforti F, Camastra S, Ferrannini E. Insulin prolongs the QTc interval in humans. American Journal of Physiology- Regulatory, Integrative and Comparative Physiology 2000;279;2022-2025

[3] Ireland RH, Robinson RT, Heller SR, Marques JL, Harris ND. Measurement of high resolution ECG QT interval during controlled euglycaemia and hypoglycaemia. Physiol Meas. 2000;21;295-303

[4] Lee S, Harris ND, Robinson RT, Yeoh L, Macdonald IA, Heller SR. Effects of adrenaline and potassium on QTc interval and QT dispersion in man. European Journal of Clinical Investigation 2003;33;93-98

[5] Meinhold J, Heise T, Rave K, Heinemann L. Electrocardiographic Changes During Insulin-Induced Hypoglycemia in Healthy Subjects. Horm Metab Res 1998;30;694-697

[6] Robinson RTCE, Harris ND, Ireland RH, Lindholm A, Heller SR. Comparative effect of human soluble insulin and insulin aspart upon hypoglycaemia-induced alterations in cardiac repolarization. British Journal of Clinical Pharmacology 2003;55;246-251

[7] Lee SP, Harris ND, Robinson RT, Davies C, Ireland R, Macdonald IA, Heller SR. Effect of atenolol on QTc interval lengthening during hypoglycaemia in type 1 diabetes. Diabetologia 2005;48;1269-1272

[8] Marques JLB, George E, Peacey SR, Harris ND, Macdonald IA, Cochrane T, Heller SR. Altered ventricular repolarization during hypoglycaemia in patients with diabetes. Diabetic Medicine 1997;14;648-654

[9] Robinson RT, Harris ND, Ireland RH, Macdonald IA, Heller SR. Changes in cardiac repolarization during clinical episodes of nocturnal hypoglycaemia in adults with Type 1 diabetes. Diabetologia 2004;47;312-315

[10] Campbell I. Dead in bed syndrome: a new manifestation of nocturnal hypoglycaemia? Diabet Med 1991;8;3-4

[11] Robinson RT, Harris ND, Ireland RH, Lee S, Newman C, Heller SR. Mechanisms of abnormal cardiac repolarization during insulin-induced hypoglycemia. Diabetes 2003;52;1469-1474

[12] DeFronzo RA, Hendler R, Christensen N. Stimulation of counterregulatory hormonal responses in diabetic man by a fall in glucose concentration. Diabetes 1980;29;125-131 